\title{
Determinantes de la distribución de Pinus spP. en la Altiplanicie Central de Chiapas, México
}

\author{
María Patrocinio Alba-López', Mario González-Espinosa ${ }^{2,4}$, Neptalí Ramírez-Marcial ${ }^{2}$ y Miguel \\ Ángel CASTILlo-SANTIAGO ${ }^{3}$
}

\author{
1 Programa de Maestría en Recursos Naturales y Desarrollo Rural, El Colegio de La Frontera Sur (ECOSUR), Apartado Postal 63, \\ 29200 San Cristóbal de Las Casas, Chiapas, México. \\ ${ }^{2}$ Departamento de Ecología y Sistemática Terrestres, División de Conservación de la Biodiversidad, ECOSUR, Apartado Postal \\ 63, 29200 San Cristóbal de Las Casas, Chiapas, México. \\ ${ }^{3}$ Laboratorio de Análisis de Información Geográfica y Estadística (LAIGE), ECOSUR, Apartado Postal 63, 29200 San Cristóbal \\ de Las Casas, Chiapas, México. \\ ${ }^{4}$ Autor para la correspondencia: Tel: (967) 678-4558, Fax: (967) 678- 4557, correo-e: mgonzale@sclc.ecosur.mx
}

\begin{abstract}
Resumen: Analizamos las relaciones de la benignidad ambiental, la estacionalidad y la calidad/fertilidad del suelo con la distribución de nueve especies de Pinus de Los Altos de Chiapas, México. Usamos información georreferenciada de 241 ejemplares herborizados colectados en 180 sitios entre 1939 y 1999. Tres componentes principales explicaron 76.5\% de la variación en la distribución de las especies. El primer componente se relacionó con las condiciones de humedad, el segundo con la temperatura y el tercero con la calidad/fertilidad del suelo. Se intentó explicar la distribución de las especies con una regresión logística. La presencia de P. maximinoi, P. oocarpa, $P$. pseudostrobus y $P$. tecunumanii se explicó con la altitud $(P<0.01)$, mientras que la evapotranspiración real anual y la calidad del suelo contribuyeron a explicar la distribución de $P$. pseudostrobus, $P$. oocarpa y $P$. montezumae $(0.05<P<0.10)$. Discutimos la pertinencia de este tipo de estudios para el manejo y conservación de este grupo de especies y otras asociadas a ellas.
\end{abstract}

Palabras clave: coníferas, pinos, regresión logística, sistemas de información geográfica, variables ambientales.

\begin{abstract}
We analyzed the relationships of habitat favorability, seasonality, and soil fertility/quality with the distribution of nine species of Pinus in the highlands of Chiapas, Mexico. We used geo-referenced information obtained from the labels of 241 herbarium vouchers collected in 180 localities between 1939 and 1999. Three principal components explained 76.5\% of total variance: a first component was related to humidity conditions, the second one to temperature, and the third one to soil quality. Logistic regression was used to explain the distribution of each species. $P$. maximinoi, $P$. oocarpa, $P$. pseudostrobus, and $P$. tecunumanii were related to elevation $(P<0.01)$; actual annual evapotranspiration and soil quality contributed to explain the distribution of $P$. pseudostrobus, $P$. oocarpa, and $P$. montezumae ( 0.05 $<P<0.10)$. We discuss some implications and the relevance of this kind of studies in supporting decisions aimed at pine management and conservation and of many other taxa associated to them.
\end{abstract}

Key words: conifers, environmental variables, geographical information systems, logistic regression, pines.

$\mathbf{M}$ éxico posee la mayor riqueza de especies del género Pinus (según diversos criterios, entre 43 y 49 de las 93-100 especies a nivel mundial) y se reconoce que la mayoría de ellas son endémicas (Mirov, 1976; Eguiluz-Piedra, 1982; Styles, 1993; Farjon y Styles, 1997; Mabberley, 1997). Las especies de Pinus participan en grado variable en el dosel de formaciones boscosas que en conjunto ocupan $c a$. $10 \%$ del territorio nacional (Palacio-Prieto et al., 2000) y que representan el principal recurso de la industria forestal del país (Challenger, 1998). En México, los bosques con dominancia de Pinus, o en asociación con Quercus (encinos o robles), se distribuyen en regiones con climas templados y fríos, lluviosos o subhúmedos, con lluvias en verano y una estación seca bien definida en invierno y primavera, con temperatura media anual de $10-20^{\circ} \mathrm{C}$ y precipitación entre 500 y >2,000 mm al año (climas $\mathrm{Cw}$ y $\mathrm{Cm}$ de acuerdo a la clasificación de Köppen modificada por García, 1987). Las asociaciones con predominio de especies de Pinus se encuentran más ampliamente distribuidas entre 1,500 y 3,000 metros de altitud (Rzedowski, 1978; Richardson, 1998). 
En Chiapas ca. $10,000 \mathrm{~km}^{2}(13.8 \%$ del territorio estatal, Palacio-Prieto et al., 2000) están cubiertos por bosques de pino y pino-encino, y constituyen así los tipos de vegetación predominantes en las tierras templadas y frías de la entidad (Sierra Madre de Chiapas y Altiplanicie Central; Miranda, 1952; Breedlove, 1981). Para el estado de Chiapas, Farjon y Styles (1997) reportan 11 especies y dos variedades en una de ellas. En décadas recientes el desmonte de bosques de encino, de pino-encino y mesófilo de montaña o de neblina, causado por actividades agrícolas y ganaderas, ha favorecido el reclutamiento, la sobrevivencia y el crecimiento de especies de Pinus (González-Espinosa et al., 1995; Ramírez-Marcial et al., 2001; Galindo-Jaimes et al., 2002). En la actualidad, las masas forestales menos alteradas en su composición original se limitan a las cimas, a las áreas de pendientes pronunciadas o a los sitios más alejados de las poblaciones humanas (Alemán-Santillán, 1989; Ochoa-Gaona y González-Espinosa, 2000).

En este trabajo conjugamos información cartográfica sobre factores ambientales con datos georreferenciados de etiquetas de ejemplares herborizados para analizar la distribución de las especies del género Pinus en la Altiplanicie Central de Chiapas. Se parte del supuesto de que las variables elegidas representan dimensiones ambientales de los nichos de las especies de Pinus en cuanto a: (1) benignidad del ambiente, (2) heterogeneidad temporal o estacionalidad, y (3) calidad/ fertilidad del suelo.

\section{Materiales y métodos}

Región de estudio. El estudio se limita al área central del estado de Chiapas localizada a una altitud de $>1,000 \mathrm{~m}$ ( $\mathrm{ca}$. $27,850 \mathrm{~km}^{2}$ ), entre $91^{\circ} 42^{\prime}$ y $93^{\circ} 18^{\prime}$ de longitud oeste, y $16^{\circ}$ $07^{\prime}$ a $17^{\circ} 32^{\prime}$ de latitud norte (figura 1 ). El clima predominante es templado subhúmedo (temperatura media anual de 13$\left.17^{\circ} \mathrm{C}\right)$, con lluvias en verano $(1,100-1,600 \mathrm{~mm}$ anuales; $>85 \%$ de la lluvia se presenta entre mayo y octubre; García, 1987; Díaz-Hernández et al., 2000) y con heladas posibles en áreas deforestadas a más de 2,000 m (no se han registrado heladas en el interior de los bosques; Romero-Nájera, 2000). Los tipos de rocas más frecuentes son calizas del Mesozoico y el Terciario Inferior y Medio, con afloramientos aislados de rocas volcánicas del Terciario Superior y el Cuaternario (Müllerried, 1957), que dan origen a suelos de tipo Rendzina, Luvisol y Acrisol de color café oscuro, con textura arcillosa o franco-arcillosa, someros a moderadamente profundos (INEGI, 1993a,b,c).

Bases de datos. Se obtuvo la información de las etiquetas de ejemplares de herbario de especies de Pinus disponibles para el estado de Chiapas en los siguientes herbarios: Academia de Ciencias de California (herbarios CAS y DS, San Francisco, California, EUA, hasta 1996), El Colegio de la Frontera Sur (ECOSUR, San Cristóbal de Las Casas, Chis., hasta 1999), MEXU (Universidad Nacional Autónoma de México,

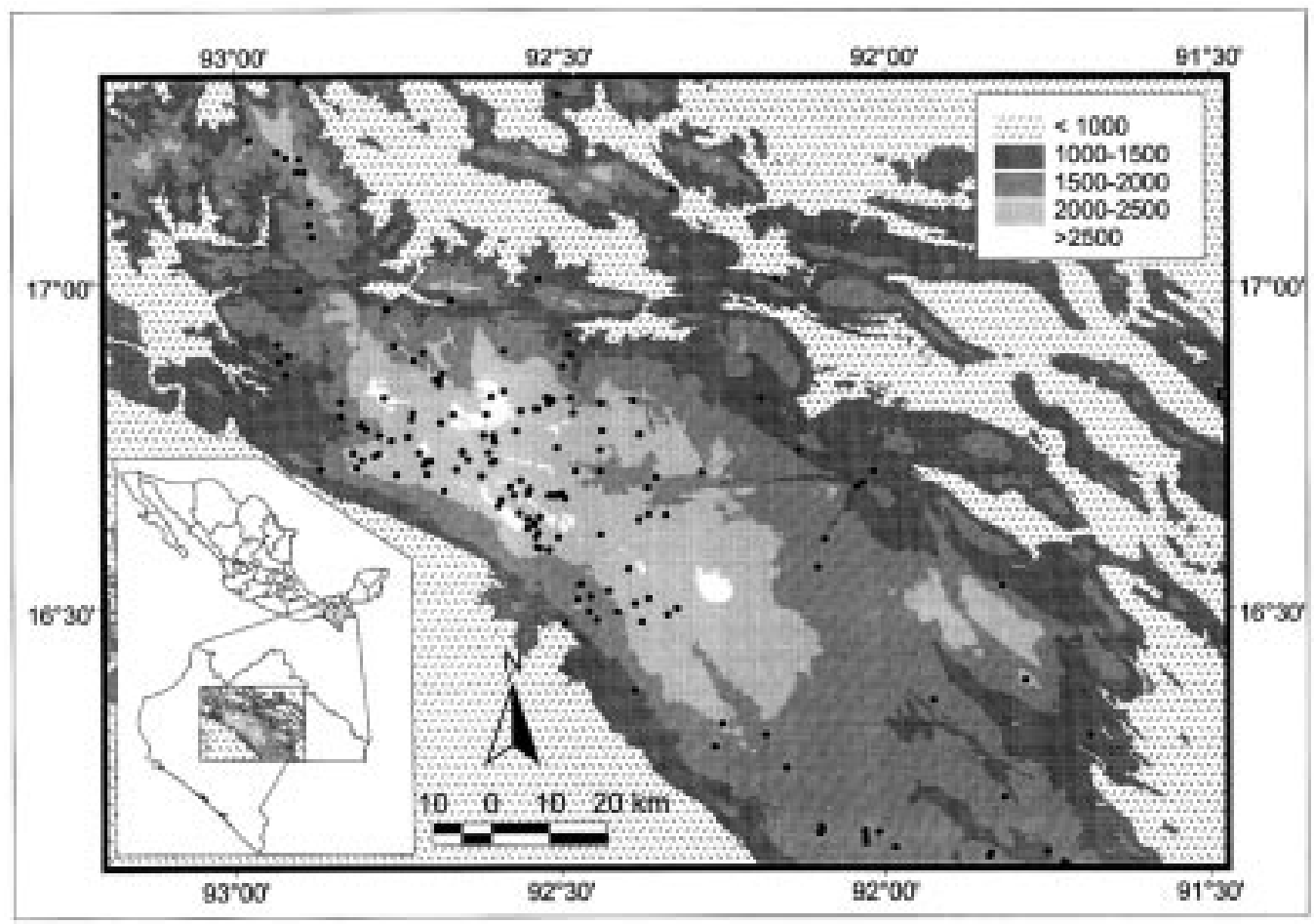

Figura 1. Ubicación del estado de Chiapas, la Altiplanicie Central y las 180 localidades en diferentes bandas altitudinales (m) en las que se han recolectado los 241 ejemplares de las nueve especies de Pinus utilizadas en este estudio. 
México, D.F., hasta 1999) y XAL (Instituto de Ecología, Xalapa, Ver., hasta 1997). De cada etiqueta se obtuvo la identidad taxonómica, el (los) colector(es), la fecha y la localidad de colecta, y cuando estuvieron disponibles, la descripción del hábitat y las coordenadas geográficas. La información taxonómica dependió de las revisiones que pudieran haber hecho los especialistas en los diferentes herbarios antes de la recopilación. No se utilizaron ejemplares sin identificación a nivel de especie.

Georreferenciación y proyección de los registros. La información sobre identidad taxonómica y localidades de colecta se obtuvo de la base de datos alfanumérica de árboles para el estado de Chiapas recopilada por M. GonzálezEspinosa y N. Ramírez-Marcial (CONABIO, proyecto F-019). Las localidades se ubicaron en cartas topográficas de INEGI 1:50,000 para asignarles coordenadas geográficas por interpolación, las cuales se proyectaron a Universal Transversa de Mercator (UTM) Zona 15, con el programa Arc/View 3.2 (ESRI, 1996).

Datos ambientales. Se realizaron sobreposiciones topológicas (espaciales) de la cartografía de INEGI (escala 1:250,000) digitalizada por el Laboratorio de Información Geográfica y Estadística (LAIGE) de El Colegio de la Frontera Sur (ECOSUR), relativa a variables climáticas (INEGI, 1984a,b,c,d,e,f), altitud (INEGI, 1988, 1992, 1994), unidades y subunidades de suelos según el sistema de clasificación de FAO-UNESCO (INEGI, 1993a,b,c) y un modelo digital de elevación (DEM). De los mapas digitalizados se obtuvieron las siguientes variables: temperaturas máximas y mínimas promedio de los períodos mayo-octubre y noviembre-abril (isotermas separadas por $3^{\circ} \mathrm{C}$ ), lluvia $(\mathrm{mm})$ entre mayo y octubre (época húmeda; isoyetas separadas cada $100 \mathrm{~mm}$ entre los valores $<1,000$ $\mathrm{mm}$, y cada $300 \mathrm{~mm}$ en los valores $>1,700 \mathrm{~mm}$ ) y entre abril y noviembre (época de secas; isoyetas cada 25-100 $\mathrm{mm})$. Con base en estas seis variables se calcularon para cada localidad de colecta: (i) temperatura media anual, (ii) temperatura máxima media anual, (iii) temperatura mínima media anual, (iv) precipitación anual, y (v) promedio de evapotranspiración real anual $\left(E T R_{A}, \mathrm{~mm}\right.$ año $\left.{ }^{-1}\right)$, calculada con la fórmula de Turc (1954), donde $E T R_{A}=P /[0.9+$ $\left.(\mathrm{P} / \mathrm{L})^{2}\right]^{1 / 2}$, y $L=300+25 T+0.005 T^{3}$. Esta variable ha sido usada en cartografía hidrológica nacional de escala 1:4,000,000 (Maderey y Torres-Ruata, 1990), está correlacionada a nivel regional con la productividad primaria (Rosenzweig, 1968) y sirve como un índice de la cantidad de energía disponible para el desarrollo vegetal en ambientes terrestres (Rosenzweig y Abramsky, 1993). Finalmente, obtuvimos estimadores de la estacionalidad ambiental a partir de (vi) la diferencia de la precipitación en época húmeda y época seca (DILLUSEC) y de (vii) la relación de proporciones de la evapotranspiración real de la época de lluvias (mayo-octubre) respecto a la de secas (noviembre-abril): $E T_{H S}=E T R_{H} / E T R_{S}$.

De las cartas edafológicas de INEGI (escala 1:250,000) se obtuvieron para cada localidad los taxones de suelo (combinaciones de unidades, subunidades y fases físicas del suelo; FAO-UNESCO, 1974). Se calculó un índice de calidad/ fertilidad de los suelos (CFS) con base en la interpretación de las propiedades físicas y químicas de los taxones señaladas en la cartografía (incluyendo clase textural y fases físicas) y en la descripción de las unidades y subunidades de suelos (FAOUNESCO, 1974). La calificación (González-Espinosa et al., sometido) se basa en los criterios de productividad de suelos sugeridos por Riquier et al. (1970), que se han utilizado en protocolos de evaluación de tierras en varios países europeos (p. ej. Porta-Casanellas et al., 1998). Asignamos a cada taxón un valor categórico (1-8, en orden creciente de contribución a la calidad/fertilidad) para cada uno de los siguientes atributos: drenaje $(D)$, profundidad efectiva $(P)$, textura/estructura $(T)$, porcentaje de saturación de bases $(V)$, contenido de materia orgánica $(O)$, reserva de minerales $(R)$ y capacidad de intercambio mineral $(A)$. Estas variables se integraron en la fórmula $D+P+T+V+O+R+A=C F S$. Así por ejemplo, a un Gleysol eútrico le correspondió el siguiente conjunto de valores $1+3+2+8+5+5+5=29$, correspondiente a un suelo muy mal drenado, poco profundo, con textura/estructura pobre, que puede tener una muy alta saturación de bases, y valores medios de materia orgánica, reservas minerales y actividad de arcillas. A un Luvisol crómico con una fase lítica profunda se le asignaron los valores $8+4+8+6+5+8+8$ $=47$, correspondientes a un suelo bien drenado, medianamente profundo, con buena textura/estructura, con valores medios de saturación de bases y materia orgánica, y con valores altos de reservas minerales y actividad de arcillas.

Análisis. Se realizó un análisis de componentes principales para todas las localidades con un total de 10 variables ambientales (altitud, precipitación en época húmeda y seca, temperaturas máxima y mínima media anual, $E T R_{H}, E T R_{S}$, $E T_{H S}, D I L L U S E C$ y $C F S$ ). Todas las variables se estandarizaron antes del análisis. Se graficó la distribución de cada especie con referencia a los dos primeros componentes (Dillon y Goldstein, 1984). Se calculó el coeficiente de correlación de Pearson $(r)$ entre las variables ambientales para apoyar la selección a priori de las que pudieran representar dimensiones ambientales independientes en modelos de regresión logística ajustados para cada especie de Pinus (Fisher y van Belle, 1993; Norûsis, 1997). La selección de las variables predictivas en cada modelo se hizo con un procedimiento de eliminación por pasos (stepwise backward selection) y se usó el estadístico de Wald para la remoción de variables (valor predeterminado de $p=0.1$ ). Los análisis estadísticos fueron computados con el paquete SPSS, versión 10.0 (SPSS, 1999) y se utilizaron los criterios predeterminados. 


\section{Resultados}

En este estudio se dispuso de 660 registros georreferenciados de especies de Pinus; sólo 36.5\% (241 registros) se encontraron determinados a nivel específico o infraespecífico, y poco más de la mitad de estos registros se encuentran depositados en ECOSUR ( ca. 59\%), seguido de CAS (29.5\%) y MEXU y XAL ( $10.8 \%$ conjuntamente). Las 241 colectas de herbario georreferenciadas corresponden a nueve especies de Pinus (nomenclatura según Farjon y Styles, 1997) recolectadas entre 1939 y 1999 en 180 localidades dentro de la región de estudio: Pinus ayacahuite C. Ehrenb. ex Schltdl. (14 ejemplares), P. devoniana Lindl. (24), P. maximinoi H.E. Moore (16), P. montezumae Lamb. (25), P. oocarpa Schiede ex. Schltdl. (32), P. pseudostrobus Lindl. (61, incluye las variedades apulcensis (Lindl.) Shaw y pseudostrobus), $P$. strobus L. var. chiapensis Martínez (21), P. tecunumanii Eguiluz et J.P.Perry (44) y $P$. teocote Schiede ex. Schltdl. et Cham. (4); en adelante no se utilizarán los pocos datos para esta especie. En conjunto, las nueve especies fueron colectadas sobre un intervalo altitudinal de $950-2,800 \mathrm{~m}$, con temperatura media anual de $11-23^{\circ} \mathrm{C}$ y un promedio de precipitación anual de 940-3,250 mm (cuadro 1). La mayoría de las colectas se han efectuado en la región centro-occidental del área de estudio a altitudes $>2000 \mathrm{~m}$ (figura 1).

El análisis de componentes principales a partir de 10 variables ambientales incluidas indicó que la distribución de Pinus está relacionada con siete variables contenidas en tres componentes ( $76.5 \%$ de la varianza total; cuadro 2). El primer componente explicó $39.9 \%$ de la varianza total y estuvo asociado de manera directa con la cantidad de lluvia durante las épocas húmeda y seca, y con la $E T R_{S}$. (cuadro 2). Un segundo componente (que explicó $26.5 \%$ adicional de la varianza total) incluyó de manera positiva a las temperaturas máxima y mínima media anual. El tercer componente explicó $10.1 \%$ adicional de la varianza y se asoció de manera directa con el índice de calidad/fertilidad de suelos (CFS). Los dos primeros componentes representaron apropiadamente algunas de las variables originales, al contribuir a la comunalidad entre 82.9 (precipitación en época seca) y $95.0 \%$ (temperatura máxima); el tercer componente representó a sólo una variable original (CFS) con $78.2 \%$ (cuadro 2). $\mathrm{Al}$ relacionar los dos primeros componentes principales con la distribución de las especies de Pinus en el área de estudio, se encontró que éstas difieren en su distribución sobre los componentes (figura 2). Al promediar los valores de ambos ejes para cada una de las especies y proyectarlos junto con los valores de las 10 variables ambientales (figura $2 \mathrm{~b}$ ), se encontró que Pinus maximinoi, $P$. strobus var. chiapensis y $P$. oocarpa presentaron mayor asociación con ambientes más húmedos, condición representada a lo largo del primer componente. Por su parte, las cinco especies restantes (Pinus ayacahuite, P. devoniana, $P$. montezuma, $P$. pseudostrobus y $P$. tecunumanii) se asociaron con ambientes más secos. Al considerar el segundo
Cuadro 1. Valores extremos y promedio ( \pm 1 error estándar) de las variables ambientales de 180 localidades en que se han recolectado 241 ejemplares de Pinus spp. en la Altiplanicie Central de Chiapas (> 1,000 $\mathrm{m}$ de altitud).

\begin{tabular}{lrrr}
\hline Variable ambiental & Mínimo & Máximo & $\begin{array}{r}\text { Promedio } \\
\pm 1 \text { E.E. }\end{array}$ \\
\hline Altitud $(\mathrm{m})$ & 950 & 2,800 & $1911 \pm 31$ \\
Temperatura media anual $\left({ }^{\circ} \mathrm{C}\right)$ & 11.3 & 22.5 & $17.0 \pm 0.19$ \\
Temperatura máxima media anual $\left({ }^{\circ} \mathrm{C}\right)$ & 18.0 & 28.5 & $22.7 \pm 0.19$ \\
Temperatura mínima media anual $\left({ }^{\circ} \mathrm{C}\right)$ & 6.0 & 16.5 & $11.4 \pm 0.20$ \\
Precipitación anual $(\mathrm{mm})$ & 938 & 3,250 & $1644 \pm 35$ \\
Precipitación en época húmeda $(\mathrm{mm})$ & 850 & 2,150 & $1341 \pm 22$ \\
Precipitación en época seca $(\mathrm{mm})$ & 88 & 1,100 & $304 \pm 15$ \\
TTR $_{A}{ }^{1}(\mathrm{~mm})$ & 610 & 1,226 & $827 \pm 9$ \\
ETR $_{H}{ }^{2}(\mathrm{~mm})$ & 614 & 1,211 & $829 \pm 9$ \\
ETR $_{S}{ }^{3}(\mathrm{~mm})$ & 92 & 775 & $281 \pm 11$ \\
ET $_{H S}{ }^{4}$ & 1.4 & 10.2 & $3.9 \pm 0.15$ \\
$\operatorname{DILLUSC}^{5}$ & 738 & 1,600 & $1,037 \pm 10$ \\
CFS $^{6}$ & 59 & 94 & $78 \pm 0.5$ \\
\end{tabular}

1 Evapotranspiración anual real.

2 Promedio de evapotranspiración real en la época húmeda.

3 Promedio de evapotranspiración real en la época seca.

4 Estacionalidad anual de la energía, $E T_{H S}=E T R_{H} / E T R_{S}$.

5 Estacionalidad de la lluvia, DILLUSEC: diferencia de lluvia entre la temporada de lluvias y secas.

6 CFS: índice de calidad/fertilidad de los suelos.

Cuadro 2. Matriz de valores propios y comunalidad de componentes principales entre las variables ambientales seleccionadas para las 180 localidades donde se han colectado 241 ejemplares de Pinus spp. en la región de estudio. En negritas se muestran para cada componente las tres variables con mayores valores. Véase la codificación de las variables en Materiales y métodos.

\begin{tabular}{|c|c|c|c|c|}
\hline \multirow[t]{2}{*}{ Variables } & \multicolumn{3}{|c|}{ Componentes } & \multirow[t]{2}{*}{ Comunalidad } \\
\hline & 1 & 2 & 3 & \\
\hline Altitud (m) & -0.197 & -0.055 & -0.419 & 0.217 \\
\hline $\begin{array}{l}\text { Precipitación en época } \\
\text { húmeda }(\mathrm{mm})\end{array}$ & 0.965 & 0.049 & -0.087 & 0.942 \\
\hline $\begin{array}{l}\text { Precipitación en época } \\
\text { seca }(\mathrm{mm})\end{array}$ & 0.906 & 0.049 & 0.074 & 0.829 \\
\hline $\begin{array}{l}\text { Temperatura máxima } \\
\text { media anual }\left({ }^{\circ} \mathrm{C}\right)\end{array}$ & -0.231 & 0.945 & 0.052 & 0.950 \\
\hline $\begin{array}{l}\text { Temperatura mínima } \\
\text { media anual }\left({ }^{\circ} \mathrm{C}\right)\end{array}$ & -0.080 & 0.957 & 0.059 & 0.926 \\
\hline $\operatorname{ETR}_{H}(\mathrm{~mm})$ & 0.482 & 0.833 & 0.018 & 0.926 \\
\hline $\operatorname{ETR}_{S}(\mathrm{~mm})$ & 0.952 & 0.048 & 0.042 & 0.911 \\
\hline$E T_{H S}$ & -0.786 & 0.331 & 0.003 & 0.727 \\
\hline DILLUSEC & 0.617 & -0.162 & -0.246 & 0.442 \\
\hline CFS & 0.065 & 0.331 & 0.867 & 0.782 \\
\hline$\%$ de la varianza explicada & 39.94 & 26.50 & 10.09 & \\
\hline$\%$ acumulado & 39.94 & 66.44 & 76.53 & \\
\hline
\end{tabular}

1 Proporción de la varianza de la variable original contenida en los componentes principales. 

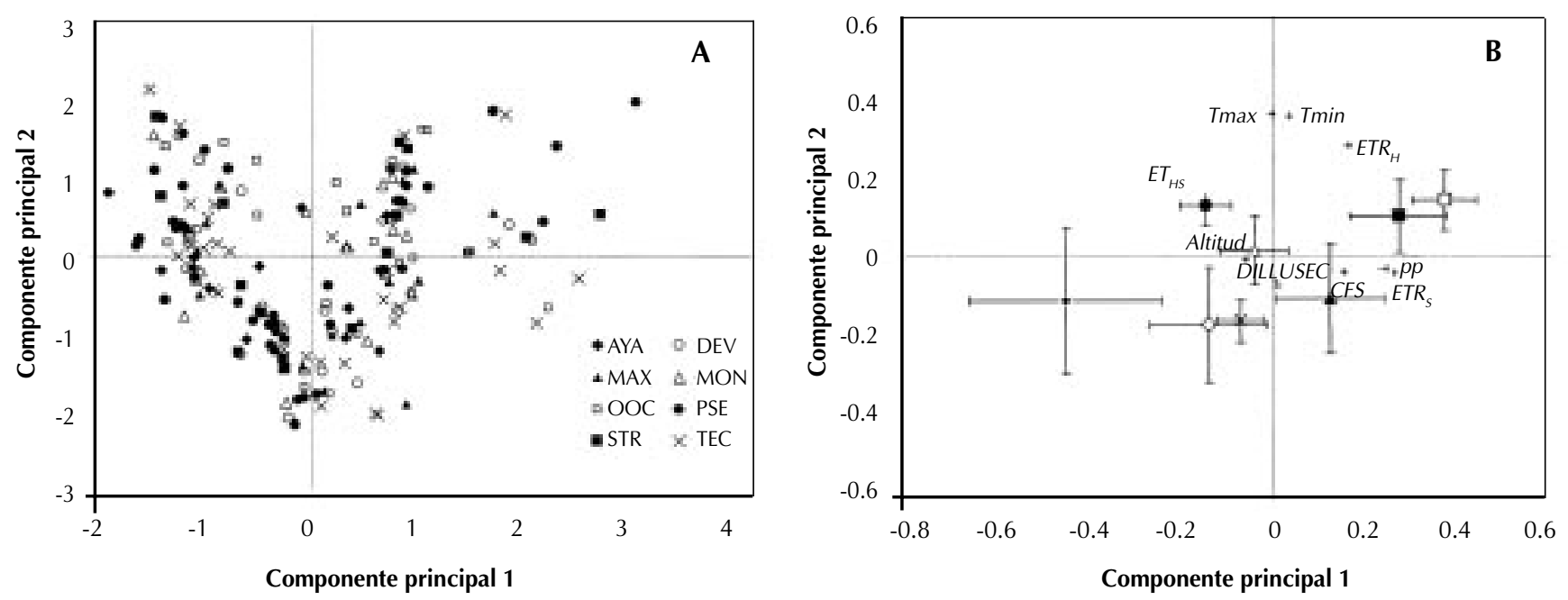

Figura 2. Distribución de las especies de Pinus en la Altiplanicie Central de Chiapas a lo largo de los dos primeros componentes principales. Se muestran (a) la totalidad de los registros para todas las especies y (b) los valores promedio ( \pm 1 E.E.) para cada una de ellas. Las cruces junto a las abreviaturas de las variables ambientales incluidas en el análisis (véase cuadro 2) representan sus valores promedio; los promedios de precipitación en época húmeda y en época seca $(p p)$ se sobreponen casi exactamente. AYA = Pinus ayacahuite, DEV $=P$. devoniana, $\mathrm{MAX}=P$. maximinoi, $\mathrm{MON}=P$. montezumae, $\mathrm{OOC}=P$. oocarpa, $\mathrm{PSE}=P$. pseudostrobus, $\mathrm{STR}=P$. strobus var. chiapensis, $\mathrm{TEC}=P$. tecunumanii.

componente, cuatro especies se asociaron con ambientes en los que la temperatura media anual alcanza valores relativamente altos ( $P$. montezumae, $P$. oocarpa, $P$. pseudostrobus y $P$. strobus var. chiapensis), mientras que las especies más fuertemente asociadas a ambientes con temperaturas relativamente bajas fueron $P$. ayacahuite, $P$. devoniana, $P$. maximinoi y $P$. tecunumanii (figura $2 \mathrm{~b}$ ).

La altitud y la $C F S$ no mostraron correlación $(P>0.05)$ con ninguna de las variables relacionadas con temperatura, precipitación, evapotranspiración o estacionalidad (cuadro 3). Sin embargo, la evapotranspiración real anual $\left(E T R_{A}\right)$ tuvo una correlación significativa $(p<0.001)$ con la lluvia anual y la temperatura media anual (cuadro 3 ). Con base en estos resultados, las variables predictivas de la distribución de cada una de las especies usadas en el ajuste de los modelos de regresión logística fueron: la altitud, el logaritmo natural de $E T R_{A}$, el logaritmo natural de $E T R_{H S}$ y el $C F S$. En general, la bondad de ajuste de los modelos para cada especie fue

Cuadro 3. Coeficientes de correlación de Pearson $(r)$ entre variables ambientales para las 180 localidades donde se han colectado 241 ejemplares de Pinus spp. en la región de estudio. $P A=$ precipitación anual, $T M_{A}=$ temperatura media anual. Véase la codificación de las restantes variables en Materiales y métodos. $\mathrm{NS}=P>0.05 ;^{*}=P \leq 0.05 ; * *=P<0.01 ; * * *=P<0.001$.

\begin{tabular}{|c|c|c|c|c|c|c|c|c|}
\hline & Altitud & $P A$ & $T M_{A}$ & $E T R_{A}$ & $E T R_{H}$ & $E T R_{S}$ & $E T_{H S}$ & DILLUSEC \\
\hline$P A$ & $-0.14 \mathrm{NS}$ & & & & & & & \\
\hline TMA & $0.05 \mathrm{NS}$ & $-0.15^{*}$ & & & & & & \\
\hline$E T R_{A}$ & $-0.04 \mathrm{NS}$ & $0.38^{* * *}$ & $0.82^{* * *}$ & & & & & \\
\hline$E_{H}$ & -014 NS & $0.50^{* * *}$ & $0.52^{* * *}$ & $0.72^{* * *}$ & & & & \\
\hline$E T R_{S}$ & $-0.12 \mathrm{NS}$ & $0.95^{* * *}$ & $-0.12 \mathrm{NS}$ & $0.40^{* * *}$ & $0.49^{* * *}$ & & & \\
\hline$E T_{H S}$ & 0.12 NS & $-0.67^{* * *}$ & $0.33^{* * *}$ & $-0.15^{*}$ & $-.010 \mathrm{NS}$ & $-0.75^{* * *}$ & & \\
\hline DILLUSEC & $-0.11 \mathrm{NS}$ & $0.59 * * *$ & $-0.05 \mathrm{NS}$ & $0.34^{* * *}$ & $0.31^{* * *}$ & $0.39 * * *$ & $-0.37^{* * *}$ & \\
\hline CFS & $-0.03 \mathrm{NS}$ & $0.05 \mathrm{NS}$ & $-0.07 \mathrm{NS}$ & $-0.04 \mathrm{NS}$ & -0.08 NS & $0.06 \mathrm{NS}$ & $-0.07 \mathrm{NS}$ & $-0.02 \mathrm{NS}$ \\
\hline
\end{tabular}


Cuadro 4. Coeficiente $(B)$, error estándar de $B$, estadístico de Wald y probabilidad asociada de las variables predictivas que mostraron significancia $(P<0.1)$ en los modelos de regresión logística ajustados para cada una de las especies de Pinus en el área de estudio. El conjunto inicial de variables incluyó altitud, $\ln E T R_{A^{\prime}} \ln E T_{H S}$ y CFS. Véase la codificación de variables en Materiales y métodos.

\begin{tabular}{|c|c|c|c|c|}
\hline & $B$ & S.E. & $\begin{array}{c}\text { Estadístico } \\
\text { de Wald }\end{array}$ & $P$ \\
\hline \multicolumn{5}{|c|}{ P. ayacahuite } \\
\hline Altitud & 0.001 & 0.001 & 3.226 & 0.072 \\
\hline \multicolumn{5}{|c|}{ P. maximinoi } \\
\hline Altitud & -0.002 & 0.001 & 14.71 & 0.000 \\
\hline \multicolumn{5}{|c|}{ P. montezumae } \\
\hline $\ln E T R_{A}$ & 2.205 & 1.158 & 3.627 & 0.057 \\
\hline \multicolumn{5}{|l|}{ P. oocarpa } \\
\hline Altitud & -0.002 & .000 & 18.509 & 0.000 \\
\hline $\ln E T R_{A}$ & 1.913 & 1.101 & 3.016 & 0.082 \\
\hline \multicolumn{5}{|c|}{ P. pseudostrobus } \\
\hline Altitud & 0.001 & 0.000 & 10.031 & 0.002 \\
\hline $\ln E T_{H S}$ & 0.498 & 0.258 & 3.719 & 0.054 \\
\hline$C F S^{H S}$ & -0.039 & 0.021 & 3.359 & 0.067 \\
\hline \multicolumn{5}{|c|}{ P. tecunumanii } \\
\hline Altitud & 0.001 & 0.000 & 8.829 & 0.003 \\
\hline
\end{tabular}

relativamente pobre (los valores de la $R^{2}$ de Nagelkerke, un estimador de la proporción de la varianza explicada, fueron $<0.14)$. La probabilidad de presencia de P. pseudostrobus, modelada a partir de las colectas realizadas a la fecha en el área de estudio, fue más alta a mayores altitudes, en ambientes con estacionalidad más acusada (mayor diferencia en la disponibilidad de energía biológicamente útil entre las temporadas húmeda y seca) y en localidades en las que predominan suelos relativamente infértiles o de baja calidad para la producción vegetal (cuadro 4). La probabilidad de presencia de $P$. oocarpa se explicó en mayor medida por el efecto inverso de la altitud y en menor grado por el efecto directo de la evapotranspiración real anual; la de $P$. montezumae se asoció con mayores valores de $E T R_{A}$ (cuadro 4). La probabilidad de presencia de $P$. tecunumanii y $P$. ayacahuite fue más alta en áreas localizadas a mayor altitud, mientras lo contrario se encontró para $P$. maximinoi. Las distribuciones de $P$. devoniana y $P$. strobus var. chiapensis no pudieron ser explicadas con ninguna de las variables predictivas incluidas en los modelos $(P>0.1$; cuadro 4$)$.

\section{Discusión}

Los ejemplares depositados en las colecciones museológicas son la referencia básica para estudios biogeográficos y sobre la distribución geográfica de las especies (Heyer et al., 1999; Funk y Richardson, 2002). A pesar de algunas limitaciones inherentes a las colecciones biológicas (p. ej. Coddington $e t$ al., 1991), éstas son reconocidas también como una fuente de información apropiada para la evaluación de la diversidad y la planeación de estrategias de su conservación a nivel regional (Lawton et al., 1998). La distribución de las localidades de colecta de Pinus spp. en la Altiplanicie Central de Chiapas es poco uniforme e incompleta para el total de la región (figura 1). No obstante que el número de ejemplares georreferenciados disponibles dista de ser adecuado para la mayoría de las especies, en este trabajo hemos supuesto que los registros utilizados podrían servir para mostrar los patrones más generales relacionados con su distribución. Los registros de Pinus utilizados en este estudio forman parte de una base de datos de información de herbario sobre todas las especies arbóreas colectadas en Chiapas, y no necesariamente proceden de inventarios ecológicos o dasonómicos que pudieran haber incluido características propias de las especies o del área de muestreo (p. ej. atributos estructurales, pendiente, exposición de la ladera, etc.).

La mayoría de los registros utilizados se refieren a localidades ubicadas en la parte centro-occidental del área de estudio (figura 1), debido posiblemente a la accesibilidad del área y a la concentración de esfuerzos de investigación florística, etnobotánica, ecológica y de inventario forestal en los últimos cuatro decenios (p. ej. Breedlove 1973, 1981; Berlin et al., 1974; Zamora y Velasco, 1977, 1978; GonzálezEspinosa et al., 1991, 1995; Santiago-Vera et al., 1997; Galindo-Jaimes et al., 2002). En general, las condiciones ambientales de las localidades donde se han colectado los ejemplares de Pinus usados en este estudio se incluyen dentro de los valores extremos que se han encontrado para el total de la Altiplanicie Central (M. González-Espinosa y N. Ramírez-Marcial, com. pers.). No obstante, es necesario considerar que la interpretación de nuestros resultados se ha de referir a una porción o muestra del total del área de distribución de la especie, así como a un conjunto de localidades que pueden no representar las condiciones ambientales que las mismas especies encuentran en un ámbito que excede los límites de Chiapas.

En este trabajo se destaca la relevancia que tienen algunas variables ambientales sobre la distribución de las especies de Pinus dentro el área de estudio, tales como la precipitación en las temporadas de lluvias y seca, la evapotranspiración real en tiempo de secas $\left(E T R_{S}\right)$, las temperaturas máximas y mínimas y la calidad/fertilidad de suelos (CFS; cuadro 2). Al analizar la distribución de las especies de Pinus en México y el resto de Norteamérica, Farjon (1996) y Richardson y Rundel (1998) sugieren que las especies del género pueden distinguirse en aquellas propias de climas templado-fríos y las tropicales. Nuestro análisis de componentes principales mostró algunas tendencias relativas a la distribución de las diferentes especies de Pinus (figura 2b): P. ayacahuite, $P$. devoniana, $P$. maximinoi y $P$. tecunumani se asocian con ambientes relativamente fríos (generalmente localizados a 
mayores altitudes), más húmedos en el caso de $P$. maximinoi. En contraste, $P$. montezumae se encontró asociada a sitios más cálidos y secos dentro del área de estudio, lo cual resulta un tanto inesperado de acuerdo a lo reconocido para esta especie en cuanto a temperatura (p. ej., Rzedowski, 1978; Eguiluz-Piedra, 1982; Calderón de Rzedowski y Rzedowski, 2001), ya que generalmente se encuentra a altitudes de más de 2,500 m. Esto puede deberse a un tamaño de muestra relativamente pequeño, o al muestreo de una gama de valores relativamente estrecha sobre los gradientes ambientales dentro del área de estudio. En el caso particular de $P$. pseudostrobus, el taxón con mayor número de registros (61), se encontró su asociación con ambientes secos y cálidos, y el modelo de regresión logística ajustado para esta especie indicó su asociación con regiones altas, marcadamente estacionales y con suelos de calidad pobre (localizados hacia la porción central y $\mathrm{SO}$ del área de estudio). Por su parte, $P$. oocarpa y $P$. strobus var. chiapensis se asocian con ambientes más cálidos y húmedos (figuras 1 y 2b), ubicándose más hacia la porción E de la Altiplanice Central la primera especie, y hacia el NO la segunda, mezclándose ambas con elementos propios de selvas húmedas y bosques mesófilos.

Para algunas de las especies estudiadas se ajustaron modelos de regresión logística en los que la altitud fue una variable explicativa significativa, y para $P$. ayacahuite, $P$. maximinoi y $P$. tecunumanii la única (cuadro 4 ). La altitud es una variable que conjuga varias otras que tienen una influencia más directa sobre los procesos ecológicos. Sin embargo, quizás debido a la amplitud relativamente pequeña de los gradientes ambientales incluidos en el área estudiada, restringida a localidades ubicadas a más $1,000 \mathrm{~m}$, la altitud no mostró correlación con ninguna de las otras variables ambientales (cuadro 3). Los modelos de regresión logística ajustados para $P$. montezumae y $P$. oocarpa sugieren que la evapotranspiración real, que se relaciona de manera directa con la temperatura y la precipitación, y de manera inversa con la estacionalidad (cuadro 3), puede contribuir a explicar su distribución, en ambos casos de manera directa.

El índice de calidad/fertilidad de suelos que utilizamos en este trabajo tampoco mostró correlación con las otras variables ambientales (cuadro 3). Por una parte, debe reconocerse que el índice calculado (CFS) no deja de ser una aproximación más bien gruesa a estas complejas propiedades de los suelos. Por otra parte, la asociación de las especies de Pinus con suelos relativamente pobres es bien conocida (Richardson y Bond, 1991; Richardson, 1998). En la Altiplanicie Central de Chiapas, Galindo-Jaimes et al. (2002) encontraron que el incremento en la importancia relativa $(>60 \%)$ de las especies de Pinus en los rodales de bosques de Pinus y Quercus con disturbio humano tradicional se asocia con suelos más compactados, menos ácidos y menos fértiles (menor capacidad de intercambio catiónico y menores porcentajes de nitrógeno y materia orgánica).

Las especies de Pinus son reconocidas como especies secundarias en áreas deforestadas por extracción forestal o desarrollo agrícola, en las que posteriormente se permite la regeneración forestal natural (p. ej. Williams, 1989; GonzálezEspinosa et al., 1991, 1995). En la Altiplanicie Central de Chiapas, los patrones tradicionales de uso del suelo practicados por la población indígena relacionados con la agricultura de milpa han dado como resultado un complejo de condiciones sucesionales en las que se efectúan diversas actividades de extracción de productos del bosque (AlemánSantillán, 1989; de Jong et al., 1999). El uso diferente que reciben las especies de Pinus respecto a las especies coexistentes de Quercus ha resultado en un incremento del área y densidad de los primeros, en rodales que anteriormente tuvieron bosques con mayor predominio de Quercus o bosques mesófilos de montaña (González-Espinosa et al., 1995; Ochoa-Gaona y González-Espinosa, 2000; RamírezMarcial et al., 2001; Galindo-Jaimes et al., 2002). El incremento local de la importancia de las especies de Pinus a expensas de los bosques mixtos más complejos ha sido denominado pinarización en México y otros países latinoamericanos. Este proceso implica un marcado empobrecimiento florístico y la modificación desfavorable de las condiciones edáficas y microclimáticas de los sitios (González-Espinosa et al., 1995; Romero-Nájera, 2000; Galindo-Jaimes et al., 2002). En general, la mayor frecuencia de especies de Pinus se asocia a temperaturas mínimas y máximas más extremas y condiciones de menor humedad (Romero-Nájera, 2000).

La utilización de las especies de Pinus es considerada como la opción más viable para el desarrollo forestal en las regiones templadas y frías de México (Challenger, 1998). Algunos patrones de uso actual del suelo pueden favorecer la expansión de Pinus spp. en regiones de bosques mixtos de alta diversidad florística, la cual puede asociarse a modificaciones en las condiciones climáticas y edáficas todavía insuficientemente conocidas. De esta manera, es necesario realizar estudios adicionales que permitan un mejor entendimiento de los determinantes de los cambios en su distribución y el desarrollo de herramientas de manejo para predecir algunas de las consecuencias de su uso.

\section{Agradecimientos}

Agradecemos a los curadores de los herbarios CAS, DS, MEXU, ECOSUR, CHAPA, XAL y ENCB por su amabilidad y acceso a las colecciones bajo su custodia. El personal de LAIGE (ECOSUR) facilitó el acceso, uso y procesamiento de información cartográfica y archivos electrónicos. Agradecemos a José M. Rey-Benayas, Jorge A. Meave y dos revisores anónimos por sus valiosos comentarios. Los Dres. Juan Gallardo-Díaz, J. David Álvarez-Solís y Luciano PoolNovelo aportaron sugerencias para el desarrollo de un índice de calidad/fertilidad de suelos. El financiamiento para este estudio fue otorgado por la Comisión Nacional para el 
Conocimiento y Uso de la Biodiversidad (CONABIO, contratos F-019 y L-329 a MGE), la Comisión Europea a través de los proyectos SUCRE (INCO Programme Framework 4, contrato No. ERBIC 18CT970146 a Adrian Newton y MGE) y BIOCORES (INCO Programme Framework 5, contrato No. ICA4-CT-2001-10095 a Adrian Newton y MGE), subsidios anuales de ECOSUR (1998-2003), y el Consejo Nacional de Ciencia y Tecnología (CONACYT) mediante una beca para estudios de postgrado a MPAL (No. Reg. 153031).

\section{Literatura citada}

Alemán-Santillán T. 1989. Los sistemas de producción forestal y agrícola de roza. En: Parra-Vázquez M.R. Ed. El subdesarrollo agrícola en Los Altos de Chiapas, pp. 83-151, Universidad Autónoma Chapingo, Chapingo, Edo. de México.

Berlin B., Breedlove D.E. y Raven P.H. 1974. Principles of Tzeltal Plant Classification: An Introduction to the Botanical Ethnography of a Mayan Speaking People of the Highland Chiapas. Academic Press, Nueva York, NY.

Breedlove D. 1973. The phytogeography and vegetation of Chiapas (Mexico). En: Graham A. Ed. Vegetation and Vegetational History of Northern Latin America, pp. 149-165, Elsevier, Amsterdam.

Breedlove D. 1981. Flora of Chiapas. Part I: Introduction to the Flora of Chiapas. California Academy of Sciences, San Francisco, CA.

Calderón de Rzedowski G. y Rzedowski J. 2001. Flora fanerogámica del Valle de México. Instituto de Ecología, A.C., Centro Regional del Bajío/Comisión Nacional para el Conocimiento y Uso de la Biodiversidad, Pátzcuaro, Michoacán.

Coddington J.A., Griswold C.E., Silva-Dávila D., Peñaranda E. y Larcher S.F. 1991. Designing and testing sampling protocols to estimate biodiversity in tropical ecosystems. En: Dudley E.C. Ed. The Unity of Evolutionary Biology, pp. 44-60, Dioscorides Press, Portland, OR.

Challenger A. 1998. Utilización y Conservación de los Ecosistemas Terrestres de México. Pasado, Presente y Futuro. Comisión Nacional Para el Conocimiento y Uso de la Biodiversidad (CONABIO), Instituto de Biología-UNAM y Agrupación Sierra Madre, A.C., México, D.F.

De Jong B.H.J., Cairns M.A., Haggerty P.K., Ramírez-Marcial N., Ochoa-Gaona S., Mendoza-Vega J., González-Espinosa M. y March-Mifsut I. 1999. Land-use change and carbon flux between 1970s and 1990s in central highlands of Chiapas, Mexico. Environmental Management 23:373-385.

Díaz-Hernández B.M., Plascencia-Vargas H., Arteaga-Ramírez R. y Vázquez-Peña M.A. 2000. Estudio y zonificación agroclimáticos en la región Los Altos de Chiapas, México. Investigaciones Geográficas 42:7-27.

Dillon W.R. y Goldstein M. 1984. Multivariate Analysis: Methods and Applications. Wiley, Nueva York, NY.

Eguiluz-Piedra T. 1982. Clima y distribución del género Pinus. Ciencia Forestal 7:30-44.

ESRI. 1996. Using Arc/View GIS: The Geographic Information System for Everyone. Enviroment Systems Research Institute, Inc., Redlands, CA.
FAO-UNESCO. 1974. Soil Map of the World, 1:5,000,000. Volume 1: Legend. United Nations Education, Scientific and Cultural Organization (UNESCO), París.

Farjon A. 1996. Biodiversity of Pinus (Pinaceae) in Mexico: speciation and paleo-endemism. Botanical Journal of Linnean Society 121:365-384.

Farjon A. y Styles B.T. 1997. Pinus (Pinaceae). Flora Neotropica, Monograph 75. The New York Botanical Garden, Bronx, NY.

Fisher L.D. y van Belle G. 1993. Biostatistics: A Methodology for the Health Sciences. Wiley, Nueva York, NY.

Funk V.A. y Richardson K.S. 2002. Systematic data in biodiversity studies: use it or lose it. Systematic Biology 51:303-316.

Galindo-Jaimes L., González-Espinosa M., Quintana-Ascencio P. y García-Barrios L.E. 2002. Tree composition and structure in disturbed stands with varying dominance by Pinus spp. in the highlands of Chiapas, Mexico. Plant Ecology 162:259-272.

García E. 1987. Modificaciones al sistema de clasificación climática de Köppen. Instituto de Geografía, Universidad Nacional Autónoma de México, México, D.F.

González-Espinosa M., Ochoa-Gaona S., Ramírez-Marcial N. y Quintana-Ascencio P.F. 1995. Current land-use trends and conservation of old-growth forest habitats in the highlands of Chiapas, Mexico. En: Wilson M.H. y Sader S.A. Eds. Conservation of Neotropical Migratory Birds in Mexico, pp. 190-198, Maine Agricultural and Forest Experiment Station, Miscellaneus Publication 727, Orono, ME.

González-Espinosa M., Quintana-Ascencio P.F., Ramírez-Marcial N. y Gaytán-Guzmán P. 1991. Secondary succession in disturbed Pinus-Quercus forests in the highlands of Chiapas, Mexico. Journal of Vegetation Science 2:351-360.

González-Espinosa M., Rey-Benayas J.M., Ramírez-Marcial N. y Huston M.A. Sometido. Tree diversity in the northern Neotropics: regional patterns in highly diverse Chiapas, Mexico.

Heyer W.R., Coddington J., Kress W.J., Acevedo P., Cole D., Erwin T.L., Meggers B.J., Pogue M.G., Thorington R.W., Vari R.P., Weitzman M.J. y Weitzman S.H. 1999. Amazonian biotic data and conservation decisions. Ciéncia e Cultura. Journal of the Brazilian Association for the Advancement of Science 51:372385.

INEGI. 1984a. Carta de efectos climáticos regionales mayo-octubre, E15-11 (Tuxtla Gutiérrez), escala 1:250,000. Instituto Nacional de Estadística, Geografía e Informática, México, D.F.

INEGI. 1984b. Carta de efectos climáticos regionales noviembreabril, E15-11 (Tuxtla Gutiérrez), escala 1:250,000. Instituto Nacional de Estadística, Geografía e Informática, México, D.F.

INEGI. 1984c. Carta de efectos climáticos regionales mayo-octubre, E15-8 (Villahermosa), escala 1:250,000. Instituto Nacional de Estadística, Geografía e Informática, México, D.F.

INEGI. 1984d. Carta de efectos climáticos regionales noviembreabril, E15-8 (Villahermosa), escala 1:250,000. Instituto Nacional de Estadística, Geografía e Informática, México, D.F.

INEGI. 1984e. Carta de efectos climáticos regionales mayo-octubre, E15-12, D15-3 (Las Margaritas), escala 1:250,000. Instituto Nacional de Estadística, Geografía e Informática, México, D.F.

INEGI. 1984f. Carta de efectos climáticos regionales noviembreabril, E15-12, D15-3 (Las Margaritas), escala 1:250,000. Instituto Nacional de Estadística, Geografía e Informática, México, D.F.

INEGI. 1988. Carta topográfica, E15-8 (Villahermosa), escala 1:250,000. Instituto Nacional de Estadística, Geografía e Informática, Aguascalientes, Ags. 
INEGI. 1992. Carta topográfica, E15-11 (Tuxtla Gutiérrez), escala 1:250,000. Instituto Nacional de Estadística, Geografía e Informática, Aguascalientes, Ags.

INEGI. 1993a. Carta edafológica, E15-11 (Tuxtla Gutiérrez), escala 1:250,000. Instituto Nacional de Estadística, Geografía e Informática, Aguascalientes, Ags.

INEGI. 1993b. Carta edafológica, E15-8 (Villahermosa), escala 1:250,000. Instituto Nacional de Estadística, Geografía e Informática, Aguascalientes, Ags.

INEGI. 1993c. Carta edafológica, E15-12, D15-3 (Las Margaritas), escala 1:250,000. Instituto Nacional de Estadística, Geografía e Informática, Aguascalientes, Ags.

INEGI. 1994. Carta topográfica, E15-12,D15-3 (Las Margaritas), escala 1:250,000. Instituto Nacional de Estadística, Geografía e Informática, Aguascalientes, Ags.

Lawton J.H., Bignell D.E., Bolton B., Bloemers G.F., Eggleton P., Hammond P.M., Hodda M., Holt R.D, Larsen T.B., Mawdlsley N.A., Stork N.E., Srivastava D.S. y Watt A.D. 1998. Biodiversity inventories, indicator taxa and effects of habitat modification in tropical forest. Nature 391:72-76.

Mabberley D.J. 1997. The Plant Book: A Portable Dictionary of the Vascular Plants, 2a. ed. Cambridge University Press, Cambridge.

Maderey L.E. y Torres-Ruata C. 1990. Carta IV.6.6. Evapotranspiración real, escala 1:4,000,000. Atlas Nacional de México, Instituto de Geografía, Universidad Nacional Autónoma de México, México, D.F.

Miranda F. 1952. La vegetación de Chiapas. Vol 1. Ediciones del Gobierno del Estado, Tuxtla Gutiérrez, Chiapas.

Mirov N.T. 1967. The genus Pinus. The Ronald Press, Nueva York, NY.

Müllerried F.K.G. 1957. Geología de Chiapas. Ediciones del Gobierno del Estado, Tuxtla Gutiérrez, Chiapas.

Norûsis M.J. 1997. SPSS Professional Statistics 7.5. SPSS Inc., Chicago, IL.

Ochoa-Gaona S. y González-Espinosa M. 2000. Land use and deforestation in the highlands of Chiapas, Mexico. Applied Geography 20:17-42.

Palacio-Prieto J.L., Bocco G., Velázquez A., Mas J.F., Takaki-Takaki F., Victoria A., Luna-González L., Gómez-Rodríguez G., LópezGarcía J., Palma M., Trejo-Vázquez I., Peralta A., Prado-Molina J., Rodríguez-Aguilar A., Mayorga-Saucedo R. y González M.F. 2000. La condición actual de los recursos forestales en México: resultados del Inventario Forestal Nacional 2000. Investigaciones Geográficas 43:183-203.

Porta-Casanellas J., López-Acevedo-Reguerín M. y Roquero de Laburu C. 1998. Edafología: para la Agricultura y el Medio Ambiente. Mundi-Prensa, Madrid.

Ramírez-Marcial N., González-Espinosa M. y Williams-Linera G.
2001. Anthropogenic disturbance and tree diversity in montane rain forest in Chiapas, Mexico. Forest Ecology and Management 154:311-326.

Riquier J., Bramao D.L. y Cornet J.P. 1970. A New System of Soil Appraisal in Terms of Actual and Potential Productivity. AGL/ TESR/60/6. Food and Agriculture Organization (FAO), Roma.

Richardson D.M. 1998. Ecology and Biogeography of Pinus. Cambridge University Press, Cambridge.

Richardson D.M. y W.J. Bond. 1991. Determinants of plant distribution: evidence from pine invasions. The American Naturalist 137:639-668.

Richardson D.M. y Rundel P.W. 1998. Ecology and biogeography of Pinus: an introduction. En: Richardson D.M. Ed. Ecology and Biogeography of Pinus, pp. 3-46, Cambridge University Press, Cambridge.

Romero-Nájera I. 2000. Estructura y condiciones microambientales en bosques pertubados de Los Altos de Chiapas, México. Tesis de Licenciatura, Facultad de Ciencias, Universidad Nacional Autónoma de México, México, D.F., 74 pp.

Rosenzweig M.L. 1968. Net primary productivity of terrestrial environments: predictions from climatological data. The American Naturalist 102:67-84.

Rosenzweig M.L. y Abramsky Z. 1993. How are diversity and productivity related? En: Ricklefs R.E y Schluter D. Eds. Species Diversity in Ecological Communities: Historical and Geographical Perspectives, pp. 52-65, University of Chicago Press, Chicago, IL.

Rzedowski J. 1978. Vegetación de México. Limusa, México, D.F.

Santiago-Vera T., Ochoa-Gaona S. y Alemán-Santillán T. 1997. Guía para Identificar Pinos de la Meseta Central de Chiapas, México. El Colegio de la Frontera Sur (ECOSUR), San Cristóbal de Las Casas, Chiapas.

SPSS. 1999. SPSS Base 10.0. Application Guide. SPSS Inc., Chicago, IL.

Styles B.T. 1993. Genus Pinus: A Mexican purview. En: Ramamoorthy T.P., Bye R., Lot A. y Fa J. Eds. Biological Diversity of Mexico: Origins and Distributions, pp. 397-420, Oxford University Press, Nueva York, NY.

Turc L. 1954. Le bilan d'eau des sols: relations entre les précipitation, l'évaporation et l'écoulement. Annales Agronomiques 5:491-596.

Williams M. 1989. Americans and Their Forests: A Historical Geography. Cambridge University Press, Cambridge.

Zamora C. y Velasco V. 1977. Pinus strobus var. chiapensis, una especie en peligro de extinción en el estado de Chiapas. Ciencia Forestal 2:3-23.

Zamora C. y Velasco V. 1978. Contribución al Estudio Ecológico de los Pinos en el Estado de Chiapas. Secretaría de Agricultura y Recursos Hidráulicos, México, D.F.

Fecha de recepción: 7 de marzo de 2003

Versión corregida: 11 de agosto de 2003

Aceptado: 21 de agosto de 2003 
